\title{
Magnetic near fields as a probe of charge transport in spatially dispersive conductors
}

\author{
H. R. Haakh and C. Henkel \\ Institut für Physik und Astronomie, Universität Potsdam, Karl-Liebknecht-Str. 24/25, 14476 Potsdam, Germany
}

Received: date / Revised version: date

\begin{abstract}
We calculate magnetic field fluctuations above a conductor with a nonlocal response (spatial dispersion) and consider a large range of distances. The cross-over from ballistic to diffusive charge transport leads to reduced noise spectrum at distances below the electronic mean free path, as compared to a local description. We also find that the mean free path provides a lower limit to the correlation (coherence) length of the near field fluctuations. The short-distance behavior is common to a wide range of materials, covering also semiconductors and superconductors. Our discussion is aimed at atom chip experiments where spin-flip transitions give access to material properties with mesoscopic spatial resolution. The results also hint at fundamental limits to the coherent operation of miniaturized atom traps and matter wave interferometers.
\end{abstract}

PACS. 42.50.Ar Photon statistics and coherence theory; 42.50.Nn Quantum optical phenomena in absorbing, amplifying, dispersive and conducting media; cooperative phenomena in quantum optical systems; 72.10.-d Theory of electronic transport; scattering mechanisms; 74.25.N- Response to electromagnetic fields; 42.50.Lc Quantum fluctuations, quantum noise, and quantum jumps

\section{Introduction}

Electromagnetic fluctuations have been playing a key role in physics ever since Planck discovered the black-body spectrum. They have universal properties at distances from a body large compared to the thermal (Wien) wavelength. In fact, the noise spectrum is telling a lot about material properties in the near field, due to the links provided by the Kirchhoff law and the fluctuation-dissipation theorem [1]. In practical applications like magnetic resonance imaging, this near-field noise is a limiting factor for detecting biological signals, for example [2]3]. In the case of a metallic body, electromagnetic fluctuations depend mainly on the conductivity and can reveal details about charge transport in the bulk. We focus here on a linear current-field relation in the general nonlocal form (spatial dispersion)

$$
j_{m}(\mathbf{r}, \omega)=\sum_{n} \int d^{3} \mathbf{r}^{\prime} \sigma_{m n}\left(\mathbf{r}, \mathbf{r}^{\prime}, \omega\right) E_{n}\left(\mathbf{r}^{\prime}, \omega\right),
$$

where the dependence on both $\mathbf{r}$ and $\mathbf{r}^{\prime}$ contains the crossover from ballistic to diffusive in the motion of charge carriers. This introduces the mean free path $\ell$ as a characteristic length scale. The conductivity (or dielectric) tensor now depends on both frequency and wave-vector in Fourier space (spatial dispersion) [4-10]. Further nonlocal effects are introduced by the details of the surface and the surface scattering of charge carriers [4,7, 10-16].

The anomalous skin effect is a famous consequence of the nonlocal bulk response. It is typically discussed in the regime of high frequencies, where the classical skin-depth $\delta(\omega)$ falls below the mean free path and does no longer describe screening of magnetic fields correctly [4, 17, 18]. Other relevant physical phenomena are Thomas-Fermi (Debye-Hückel) screening and Landau damping [19], connected to plasma screening due to mobile charges in the metal and electron-hole generation (internal photo effect), respectively. One can generally expect a reduction of field fluctuations close to the surface of a nonlocal metal as compared to local theory, because the bulk fields are better screened and escape less easily into the surrounding space. The nonlocal response of surfaces has also been discussed for other observables outside the range of the anomalous skin effect. Its implications have been worked out for the dispersion relation of surface plasmon modes [13 16], the transfer of heat via near field radiation [20,21], the van der Waals interaction across a electrolyte [22,23], and the Casimir interaction between two metallic half-spaces [24-26].

A motivation for this paper is the observation that spin-flip transitions of ultracold atoms held in miniaturized chip-based magnetic traps (atom chips) are sensitive to magnetic fluctuations in the near field of a metal [27-30]. Here, atoms are probing surface properties at somewhat exotic frequencies in the radio or microwave band, much below the visible to ultraviolet frequency range of conventional metal spectroscopy. (Note, however, that the microwave band is routinely used in superconductor experiments.) At the same time, the corresponding wavelengths are in the micron range because atoms illuminate the surface with their near field. Modes characterized by these frequencies and wave vectors (parallel to the surface) lie in the evanescent sector, way below the light cone; in particular their in-plane wavevector $p$ is not restricted as in propagat- 
ing vacuum fields. Therefore values $p \sim 1 / \ell$ cannot be excluded where spatial dispersion is clearly relevant. Previous work [31-34] has considered spin-flip transitions for the microtrap scenario in the local limit, identifying them as relevant challenges to miniaturization below the micron scale. We also mention the results of Ref. [35] on nuclear spin relaxation that are reproduced and generalized here, including the skin effect and covering a wider range of distances.

Another motivation is the study of spatial correlations of thermal near field radiation. These differ strongly from the blackbody limit where the wavelength provides a universal correlation (or coherence) length [36]. For an overview on the coherence of thermal radiation see Ref. [37]. Electric field correlations were discussed previously in Refs. [38-40] for homogeneous media and in Refs. [41-46] for the near field of bodies. Surface charge and current correlations have been studied in the high-temperature limit in Refs. [23, 47, 48]. In the electric case, the mean free path $\ell$ did not emerge as a characteristic length scale, neither in the distance dependence of the noise spectrum nor in the spatial autocorrelation function [44]. This may be related to sum rules and efficient screening at the surface. The magnetic field behaves differently because at distances around the mean free path there is a crossover in the noise spectrum [35]. We show here that the field correlations in a plane parallel to the metal surface become more coherent at short distances $(z \ll \ell)$, and that the correlation length involves the mean free path.

This work is organized as follows. In Section 2 we outline the calculation of magnetic spectra and spin-flip rates and give an overview on the length scales and effects that have an impact on these quantities in a system with nonlocality. Then, a specific nonlocal model for the bulk and surface response is introduced and used to obtain the near field asymptotes. We also briefly consider materials other than metals. In Sec. 3 field correlation functions above local and nonlocal metals are analyzed with respect to their correlation length. Sec. 4 summarizes the main results and reflects on their relevance for experimental setups. Two Appendices give details on the electromagnetic Green's tensor near a surface and the calculation of the nonlocal reflection coefficients.

\section{Magnetic noise and spin-flip losses}

\subsection{Noise spectra and spin flips}

Our main quantity of interest is $S_{i j}^{B}(\mathbf{r}, \omega)$, the spectral density per unit frequency of the magnetic field cross-correlation $(i, j=x, y, z)$. This spectrum can be calculated with Green's function techniques, as outlined in Appendix A. According to the fluctuation-dissipation theorem,

$$
S_{i j}^{B}(\mathbf{r}, \omega)=2 \hbar \bar{n}(\omega) \operatorname{Im} \mathcal{H}_{i j}(\mathbf{r}, \mathbf{r}, \omega) .
$$

where $\bar{n}(\omega)$ is the Bose-Einstein distribution and $\mathcal{H}_{i j}$ the magnetic Green's tensor defined in Eq. (30).

Recall that the Green's tensor gives the field radiated by a pointlike dipole source. In the presence of a surface, it therefore splits in two terms, the first one being the same as in free space [Eq. [31]], the second one describing the magnetic field reflected from the surface. In the situations considered here, the latter term dominates the spectrum [49]. For example, at a frequency of $100 \mathrm{MHz}$ and at room temperature, the spectrum of a surface at a distance of $1 \mu \mathrm{m}$ exceeds the black body spectrum by 15 orders of magnitude. The free space term in $\mathcal{H}$ can therefore be safely neglected and it is sufficient to consider the reflected Green's tensor, which is conveniently expressed in the Weyl representation as a two-dimensional Fourier integral

$$
\begin{aligned}
\mathcal{H}_{i j}(z, \omega)= & \frac{\mu_{0}}{8 \pi} \int_{0}^{\infty} d p p \kappa\left[\left(r_{\mathrm{s}}(\omega, p)+\frac{\omega^{2}}{c^{2} \kappa^{2}} r_{\mathrm{p}}(\omega, p)\right) \times\right. \\
& \left.\times\left[\delta_{i j}-\hat{z}_{i} \hat{z}_{j}\right]+2 \frac{p^{2}}{\kappa^{2}} r_{\mathrm{s}}(\omega, p) \hat{z}_{i} \hat{z}_{j}\right] e^{-2 \kappa z}
\end{aligned}
$$

Here, $\mathrm{s}$ and $\mathrm{p}$ label the two principal polarizations, $\kappa^{2}=p^{2}-$ $\omega^{2} / c^{2}$ is the propagation constant in vacuum, and $\hat{\mathbf{z}}$ the unit normal to the surface. Details on the reflection coefficients $r_{\mathrm{s}}$, $r_{\mathrm{p}}$ are given in Appendix A

Most of this work will consider near field noise, where large values of the perpendicular wave vector $p \gg \omega / c$ (evanescent waves) dominate the response and nonlocal effects become relevant. In this regime, the p-polarization involving $r_{\mathrm{p}}$ is suppressed by the prefactor $\omega^{2} /(c \kappa)^{2}$ in the integrand of Eq. (3). The analysis can thus be restricted to s-polarization for our purposes (magnetic field vector in the plane of incidence).

The magnetic noise spectrum has been measured via the loss rate of atoms from modern chip-based atom traps [27,-30]. An expression for the atomic transition rate due to fluctuations of the magnetic field can be obtained from Fermi's Golden Rule [28] or a master equation approach [32,34]

$$
\Gamma_{a \rightarrow b}(\mathbf{r})=\sum_{i, j} \frac{\mu_{i}^{a b} \mu_{j}^{b a}}{\hbar^{2}} S_{i j}^{B}\left(\mathbf{r},-\omega_{a b}\right) .
$$

Here $a(b)$ labels a magnetic sublevel that is trapped (not trapped) in the static magnetic field of the atom chip, $\mu_{i}^{a b}=\left\langle a\left|\mu_{i}\right| b\right\rangle$ is the matrix element of the magnetic dipole operator, and $\omega_{a b}$ the resonant Bohr frequency. For magnetic moments in the order of a Bohr magneton $\mu_{B}$, the prefactor in Eq. (4) translates a spectrum of $130 \mathrm{pT}^{2} / \mathrm{Hz}$ to a transition rate of one per second. Rates in this low range have been measured near conducting surfaces using ultracold atoms as a probe [33,50,51]. The magnetic near-field noise flips the spin of trapped atoms, leading to loss from a magnetic trap and setting a fundamental limit to the coherence in these setups [27,28]. Conversely, this process offers a way of probing material properties.

For atoms trapped in their electronic ground state, the magnetic moment is dominated by the contribution of the electron spin. We evaluate the matrix elements in Eq. (4) for simplicity by ignoring the quantum numbers of the nuclear spin. We can then consider a two-level system of which one state is magnetically trapped. For a static trapping field in the $x z$-plane that is tilted by an angle $\theta$ relative to the surface normal $\hat{\mathbf{z}}$, the magnetic dipole matrix elements read [32,52]

$$
\boldsymbol{\mu}^{g e}=\frac{\mu_{B} g_{S}}{2}(\cos \theta,-\mathrm{i}, \sin \theta)^{T} .
$$

Above a planar surface, the noise correlations are diagonal [see Eq. (6) below] so that the spin-flip rate is proportional to $2+$ $\sin ^{2} \theta$. The more general case including hyperfine structure can be found in Ref. [32]. 


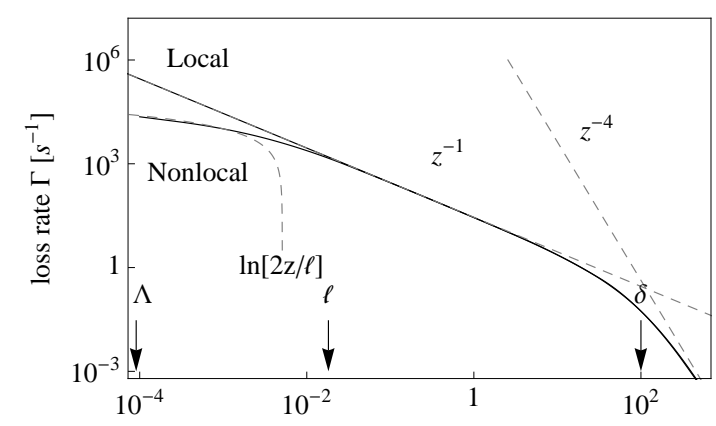

a)

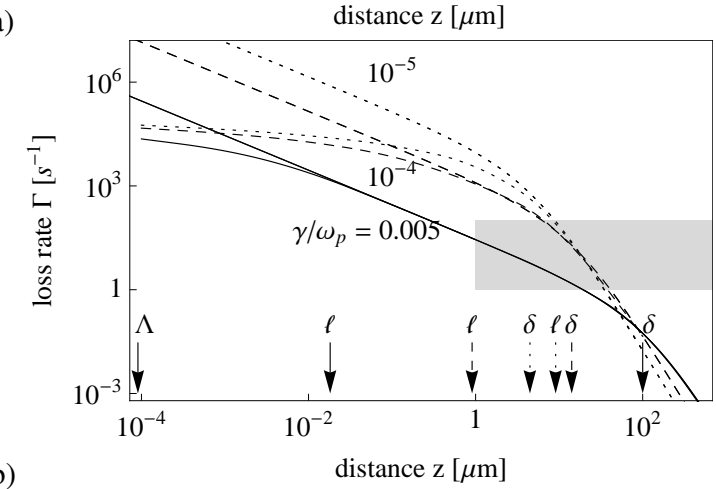

Figure 1. a) Spin flip (loss) rate near a conducting half-space described by the nonlocal Boltzmann-Mermin conductivity (8 11). The local description [Drude conductivity [12] ] and the asymptotic expressions of Eq. (6) (dashed) are shown for comparison. The length scales $\Lambda=v_{F} / \omega_{p}, \ell=v_{F} / \gamma$, and $\delta$ [Eq. (7)] illustrate the Thomas-Fermi screening length, the mean free path and the skin depth. The parameters are for gold at $T=300 \mathrm{~K}\left(\sigma=2.5 \times 10^{7} \Omega^{-1} \mathrm{~m}^{-1}, \ell=18 \mathrm{~nm}\right.$, $\gamma=6.7 \times 10^{13} \mathrm{~s}^{-1}$ ) and the surface impedance is calculated with a specular boundary condition [Eqs. 35 36] ]. Spin flips are driven by fields at the Larmor frequency $\omega / 2 \pi=1 \mathrm{MHz}$ and oriented parallel to the surface $[\theta=0$ in Eq. [5] ]. Losses due to the free space black body spectrum are much smaller and not visible on this scale.

b) Loss rates near gold surfaces with different purities. We vary the ratio $\gamma / \omega_{p}$ between relaxation rate and plasma frequency in the conductivity. The lowest curve coincides with Fig. (17). Note how the in termediate regime $\ell \ll z \ll \delta$ opens up in the dirty limit. The leftmost arrow marks the Thomas-Fermi screening length $\Lambda=v_{F} / \omega_{p}$, while $\delta$ is the normal skin depth $(7)$.

\subsection{Overview: near-field noise}

Nonlocal effects can be expected to become visible on a length scale in the order of the mean free path $\ell$ of ballistic transport of charge carriers, as was already conjectured by Rytov and coworkers [53]. Numerical calculations of the spin-flip rates for neutral atoms near a metal surface with and without a nonlocal response are shown in Fig.(1) and b). Clearly, there are three different asymptotic regimes of the distance between the atom and the surface, two of which involve distances much larger than $\ell$, where the surface spectrum cannot be distinguished from a local one. We shall find that in these regimes, the Green's tensor can be approximated by the scaling laws

$$
\begin{aligned}
& \operatorname{Im} \mathcal{H}_{i j}(\omega, z)= \\
& \left\{\begin{array}{cl}
\frac{3 \mu_{0} \delta(\omega)}{64 \pi z^{4}}\left[\delta_{i j}+\hat{z}_{i} \hat{z}_{j}\right], & \delta(\omega) \ll z \\
\frac{\mu_{0}}{32 \pi \delta^{2}(\omega) z}\left[\delta_{i j}+\hat{z}_{i} \hat{z}_{j}\right], & \ell \ll z \ll \delta(\omega)(6) \\
\frac{\mu_{0}\left[\delta_{i j}+\hat{z}_{i} \hat{z}_{j}\right]}{8 \pi \delta^{2}(\omega) \ell}\left(\ln \left[\frac{\ell}{2 z}\right]-0.077\right), & z \ll \ell .
\end{array}\right.
\end{aligned}
$$

Here, the skin depth of the normal skin effect is given by

$$
\delta(\omega)=\sqrt{\frac{2}{\mu_{0} \sigma \omega}},
$$

where $\sigma$ is the local limit of the DC conductivity [Eq. (12) below]. The local regime [first two lines in Eq. 60] were given already in Refs. [31,32].

To make this qualitative behavior understandable, we propose an interpretation in terms of an active surface volume:

i) When $z \gg \delta(\omega)$, the normal skin effect screens noise from deep in the bulk so that only a skin layer of thickness $\delta(\omega)$ contributes to the noise. The noise is proportional to the squared non-retarded fields $\sim 1 / r^{3}$ of current loops, integrated over the surface - this explains the power law $1 / z^{4}$ and the proportionality to the skin depth $\delta(\omega)$ in Eq. (6), upper line.

ii) At smaller distances $\ell \ll z \ll \delta(\omega)$, a medium-filled half-sphere of radius $\sim z$ effectively contributes to the noise. In addition, the probe particle now resolves individual current elements rather than loops. The noise then arises from the squared fields of these current elements $\left(\sim 1 / r^{2}\right)$, integrated over the volume of the half-sphere, as explained in Ref. [54].

The previous cases i) and ii) have been observed experimentally in the $\mathrm{kHz}$ to $\mathrm{MHz}$ range with sensitive magnetometers [55] and with trapped ultracold atoms [50]. In this paper, we address the regime

iii) of the extreme near field in a nonlocal conductor, $z \ll$ $\ell$ : the ballistic (rather than diffuse) motion of charge carriers creates spatial correlations in the fluctuating current field. This reduces the number of mutually uncorrelated volume elements in the half-sphere introduced in ii) above, and hence lowers the noise power. Note that the limiting value in Eq. (6) scales with $1 /\left(\delta^{2} \ell\right)$ which is actually independent of the relaxation time in a Drude conductor. The magnetic noise is related to Landau damping, or equivalently to the thermal excitation of electronhole pairs [10]. This regime is therefore quite universal, and we show in Secs. 2.5 and 2.6 that semiconductors and even superconductors follow the same scaling law.

The rest of this section will review the nonlocal response functions of the conductor and present calculations that confirm these arguments.

\subsection{Model of a nonlocal metal}

In the nonlocal regime, the current-field relationship of the bulk, i.e. the conductivity or the dielectric tensor, depends on the wave vector and it is necessary to distinguish longitudinal and transverse response functions. 


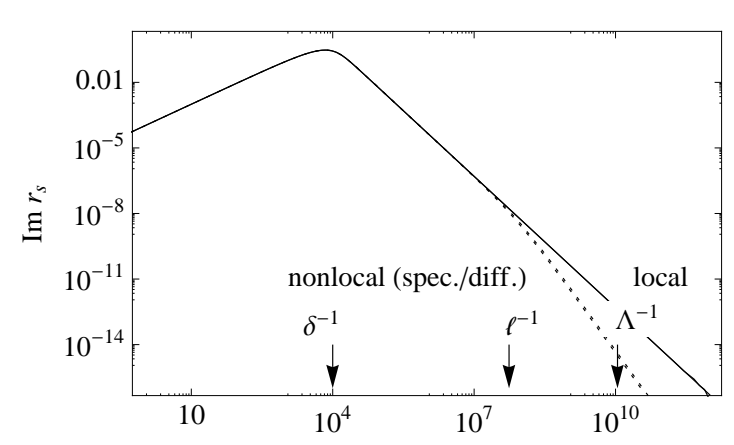

a)

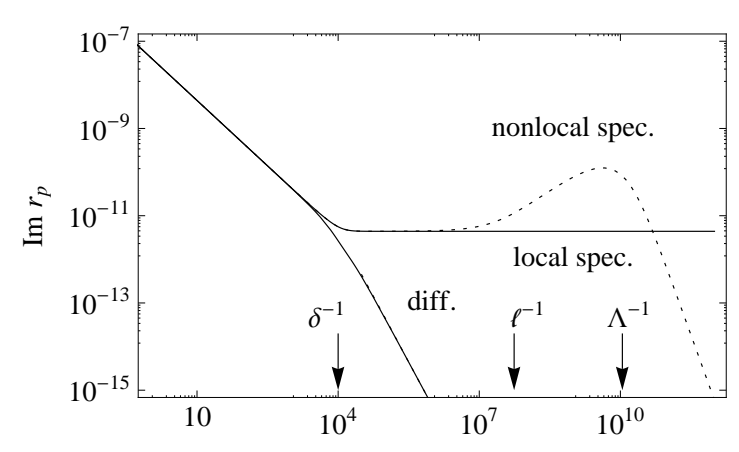

b)

wave vector $\mathrm{p}\left[\mathrm{m}^{-1}\right]$

Figure 2. Reflection coefficients $\operatorname{Im} r_{s, p}$ vs. wave vector in the evanescent sector. a) s-polarization and b) p-polarization. The curves show specular and diffuse scattering at the boundary, and bulk responses given by the local Drude model (solid), and in the nonlocal Boltzmann-Mermin model (dotted). Parameters are for gold ( $\sigma=$ $\left.2.5 \times 10^{7} \Omega^{-1} \mathrm{~m}^{-1}, \ell=18 \mathrm{~nm}, \gamma=6.7 \times 10^{13} \mathrm{~s}^{-1}\right)$. Frequency $\omega / 2 \pi=1 \mathrm{MHz}$.

Very basic descriptions taking into account some nonlocal effects are hydrodynamic models, see, e.g., Ref. [13,56]. These approaches are valid in a restricted momentum range. The simplest model does not lead to any change in the s-polarization with respect to the local limit, unless some some phenomenological transverse response is introduced. A more substantial description of a metal is given by the Boltzmann-Mermin (BM) model [6, 9, 10]

$$
\begin{aligned}
\sigma_{l}(\omega, \mathbf{k}) & =-\frac{\gamma \sigma}{\gamma-\mathrm{i} \omega} \frac{3 \omega u^{2} f_{l}(0, u)}{\omega+i \gamma f_{l}(0, u)} \\
\sigma_{t}(\omega, \mathbf{k}) & =\frac{\gamma \sigma}{\gamma-\mathrm{i} \omega} f_{t}(0, u)
\end{aligned}
$$

where $\sigma$ is the DC conductivity in the local limit, $\gamma$ describes the broadening of the electronic states at the Fermi level due to scattering. The variable $u$ contains the momentum dependence via the dimensionless functions

$$
\begin{aligned}
& f_{l}(0, u)=1-\frac{u}{2} \ln \left[\frac{u+1}{u-1}\right], \quad u=\frac{\omega+i \gamma}{k v_{F}} \\
& f_{t}(0, u)=\frac{3}{2} u^{2}-\frac{3}{4} u\left(u^{2}-1\right) \ln \left[\frac{u+1}{u-1}\right],
\end{aligned}
$$

where $v_{F}$ is the Fermi velocity. and the logarithm is taken with a branch cut along the negative real axis. These expressions are obtained in the limit $k / 2 k_{F} \ll 1$ from a more general model due to Lindhard [10], hence the redundant first argument 0 . This assumption is reasonable because for our purposes, the relevant wave vectors are in the range $k \sim 1 / z$, much smaller than the Fermi momentum $k_{F} \approx 10^{10} \mathrm{~m}^{-1}$.

The relevant frequencies for magnetic transitions, typically in the rf- to microwave range, lie in the Hagen-Rubens regime $\omega \ll \gamma \ll \omega_{p}$ where $\omega_{p}$ is the conductor's plasma frequency. In this case, spatial dispersion is obviously encoded by the parameter $u \approx \mathrm{i} / k \ell$, and the mean free path $\ell=v_{F} / \gamma$ sets the relevant scale. In the local limit $k \ell \ll 1$, both conductivities reduce to the Drude form

$$
\sigma_{l, t}(\omega, \mathbf{k}) \rightarrow \sigma(\omega)=\frac{\omega_{p}^{2} \varepsilon_{0}}{\gamma-\mathrm{i} \omega}
$$

This is the regime of the normal skin effect where Eq. (7) applies.

While the conductivity describes the bulk response of the conductor, the specific properties of the surface have an impact on the nonlocality of response, too. The calculation of the reflectivities requires the solution of the electromagnetic scattering problem at the surface. If the bulk conductivity depends on the wave vector, Fresnel's equations do not hold any more, and one has to introduce additional boundary conditions for the current density at the inner surface. The latter are modeling the way charge carriers are scattered there.

The simplest assumption is that of specular reflection of charge carriers [4, 7, 10, 13]. Diffuse scattering [11, 13] or a general combination of both mechanisms can be included, but must be treated with care to ensure that charge conservation holds at the surface [13]. Severe as it may be, this problem only occurs when electric fields have components perpendicular to the surface, i.e. in the p-polarization, while the nonlocal effects considered in this work involve the s-polarization. In addition, it is well known that the scattering mechanisms give little differences for the anomalous skin effect [4]. Much larger corrections occur, e.g. due to surface roughness [57].

The calculation of reflection coefficients at the surface of a nonlocal metal is described in Appendix B. The resulting reflection amplitudes are shown in Fig.2 for both polarizations. We plot the absorption $\operatorname{Im} r_{s, p}$ which is proportional, by reciprocity, to the radiated noise power. In the s-polarization, all models converge to the local scenario as $p \ll 1 / \ell$, as expected. Spatial dispersion leads to reduced noise for wave vectors $p \gg 1 / \ell$. The impact of nonlocality is much more important in p-polarization. The increase of p-polarized absorption in the range $1 / \ell<p<1 / \Lambda$ has been discussed previously [10, 21, 44, 58]; it is due to the internal photo-effect (creation of particle-hole pairs, Landau damping). The results with the diffuse boundary condition introduced in Ref. [12] deviate from the local limit already in the range $p \gtrsim 1 / \delta$, and become independent of the bulk conductivity. This is likely to be an artifact due to the violation of charge conservation, as discussed in Refs. [12, 14].

Note that the factor $(\omega / c \kappa)^{2} \sim 10^{-16}$ is very small where the p-polarized absorption peaks so that it is a good approximation to neglect this part in the Green's function (3). This polarization is essential, on the contrary, for situations sensitive to surface charges and electric surface fields [13], such as 
heat transport [20], heating of trapped ions [32] or the electric dipole contribution to dispersion forces [59].

\subsection{Short-distance approximation}

We derive here the asymptotic form of Eq. 6, third line. Within the approximations introduced above, the Green tensor (3) can be calculated from

$$
\mathcal{H}_{i j}(z, \omega) \approx \frac{\mu_{0}\left[\delta_{i j}+\hat{z}_{i} \hat{z}_{j}\right]}{8 \pi} \int_{0}^{\infty} \mathrm{d} p p^{2} r_{\mathrm{s}}(\omega, p) \mathrm{e}^{-2 p z}
$$

The distance range is now $z \gg \ell$ so that the relevant wave vector range is $p \sim 1 / z \gg 1 / \ell$. We start from an expansion of the Boltzmann-Mermin model (8)-(9) at small values of $u$. The limiting form of the reflection coefficients for specular scattering of charge carriers is found as (see Appendix B)

$$
p \gg 1 / \ell, \text { spec. scatt.: } \quad \operatorname{Im} r_{\mathrm{s}}(p, \omega) \approx \frac{1}{p^{3} \delta^{2}(\omega) \ell}
$$

while the diffuse boundary condition yields a result smaller by a factor $3 / 4$. This is similar to the findings of Reuter and Sondheimer [4] for the anomalous skin effect. The following analysis assumes specular scattering. The power law of Eq. (14) illustrates the reduction of noise by spatial dispersion (the decay with momentum $p$ is faster) and agrees well with a numerical calculation, as illustrated in Fig. 2 .

We split the integration range at $p \sim 1 / \ell$ and replace for $p \gg 1 / \ell$ the reflection coefficient $r_{\mathrm{s}}(\omega, p)$ by the nonlocal approximation (14). The integral then gives

$$
\begin{aligned}
& \int_{1 / \ell}^{\infty} \mathrm{d} p p^{2} r_{\mathrm{s}}(\omega, p) \mathrm{e}^{-2 p z} \approx \frac{1}{\delta^{2}(\omega) \ell} \int_{1 / \ell}^{\infty} \frac{\mathrm{d} p}{p} e^{-2 p z} \\
& =\frac{\mathrm{E}_{1}(2 z / \ell)}{\delta^{2}(\omega) \ell} \approx \frac{\ln (\ell / 2 z)-\gamma_{E}}{\delta^{2}(\omega) \ell} .
\end{aligned}
$$

Here, $\mathrm{E}_{1}(z)=\int_{z}^{\infty} \mathrm{d} t t^{-1} e^{-z t}$ is the exponential integral and $\gamma_{E} \approx 0.577$ is the Euler-Mascheroni constant. In the range $p \ll 1 / \ell$, the reflection coefficient $r_{\mathrm{s}}(\omega, p)$ is approximately equal to its local form [Eqs. 32, 33] ]. The integral then gives (we assume $\ell \ll \delta$ )

$$
\int_{0}^{1 / \ell} p^{2} d p \operatorname{Im} r_{\mathrm{s}}(\omega, p) e^{-2 p z} \approx \frac{1+\mathcal{O}(\ell / \delta)}{2 \delta^{2}(\omega) \ell} .
$$

Summing the two contributions gives the approximate Green's tensor (always for $z \ll \ell$ )

$$
\operatorname{Im} \mathcal{H}_{i j}(z, \omega) \approx \frac{\mu_{0}\left[\delta_{i j}+\hat{z}_{i} \hat{z}_{j}\right]}{8 \pi \delta^{2}(\omega) \ell}\left(\ln \left[\frac{\ell}{2 z}\right]-\gamma_{E}+\frac{1}{2}\right)
$$

This is the third regime of the Green's tensor (6) discussed in Sec.2.2 and corroborates the statement that a nonlocal description predicts less noise at short distances compared to a local one. We have thus generalized a similar result reported in Ref. [35] within the context of nuclear spin relaxation, where the normal skin effect was neglected. We conclude that for the miniaturization of atom chip experiments, a large mean free path is advantageous. Crystalline metals may push $\ell$ into a range that is achievable with atom chip traps. The other possibility may be chips based on pure semiconductor substrates that we discuss now.

\subsection{Semiconductors}

The previous analysis can be generalized to other classes of conducting materials. Strongly doped semiconductors (where the electron gas is degenerate like in a metal) may be described by the BM model (8)-(11) with modifications only in the values of the parameters. For frequencies well below the gap, a background dielectric constant $\varepsilon_{b}$ appears due to the static interband polarizability, but this does not play a role for magnetic near field noise. Weakly doped semiconductors (non-degenerate electron gas), on the other hand, are not ruled by Fermi statistics, but by a thermal distribution with the characteristic velocity $v_{T}=\left(3 k_{B} T / m\right)^{1 / 2}$ taking over the role of the Fermi velocity $v_{F}$. Here, Eqs. [10] and (11) should be replaced by [60,61]

$$
f_{t}(0, u)=1-\frac{3}{2} f_{l}(0, u)=-\frac{u}{\sqrt{\pi}} \int_{-\infty}^{\infty} d z \frac{\exp \left(-z^{2}\right)}{z-u}
$$

We observe that this is numerically very close to Eqs. (10), (11) in the local regime $k \ell \ll 1$ and differs only by a numerical factor in the deeply nonlocal regime. Up to this changed prefactor, the preceding calculations for the $r_{\mathrm{s}}$ coefficient and the Green's tensor carry through so that the physics is qualitatively the same.

Let us consider typical numbers that can be found from experiments on charge transport in silicon [62]. An n-type semiconductor with a rather low doping of $3 \times 10^{16} \mathrm{~cm}^{-3}$ is characterized by a skin depth $\delta>1 \mathrm{~cm}$ in the $\mathrm{MHz}$ range. At room temperature, $v_{T}=1.2 \times 10^{5} \mathrm{~m} / \mathrm{s}$ and $\ell \approx 60 \mathrm{~nm}$, quite comparable to the value for gold. Since it is proportional to the DC conductivity, the magnetic near field spectrum is smaller by orders of magnitude compared to a metal. Cooling the sample down to $100 \mathrm{~K}$ reduces phonon excitations and enhances the conductivity. Yet, the thermal velocity drops also to $v_{T}=$ $6.7 \times 10^{4} \mathrm{~m} / \mathrm{s}$, so that the mean free path is barely larger, $\ell \approx 85 \mathrm{~nm}$. At even lower temperatures the conduction band occupation freezes out and the response becomes local.

\subsection{Superconductors}

The nonlocal response of superconductors in the microwave range was discussed in Refs. [64, 65]. It was argued by Rickayzen [66] that screening in a superconductor does not differ greatly from a normal metal. This is because all charge carriers contribute to screening, while the specific properties of a superconductor are determined by the states close to the Fermi level. We thus expect the noise spectrum to be characterized by the same logarithmic asymptote 15 found earlier. This is indeed confirmed by numerical calculations of magnetic noise near a niobium surface, the results of which are shown in Fig. 3. We have evaluated the non-local BCS conductivity within 


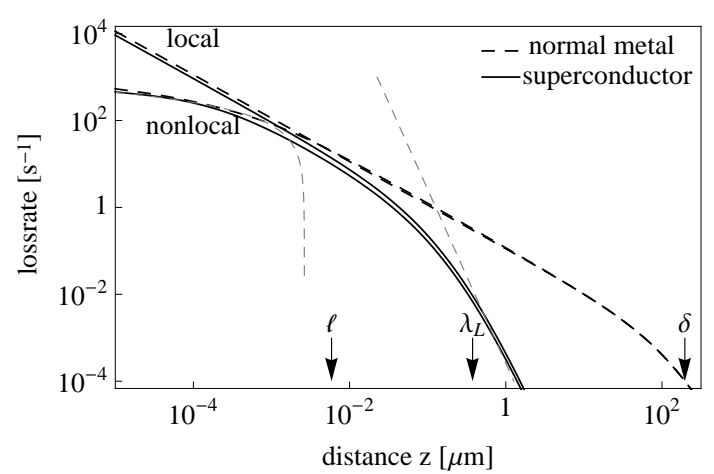

Figure 3. Spin flip (loss) rate near a surface made of superconducting niobium (solid curves) and a fictitious normal metal (dashed curves). Nonlocal effects are given for both cases as compared to the local limit. Parameters for niobium at $T=0.5 T_{c}$ follow Ref. [63] $\omega_{p}=1.33 \times 10^{16} \mathrm{~s}^{-1}, \gamma=2.38 \times 10^{-14} \mathrm{~s}^{-1}, T_{c}=9.2 \mathrm{~K}, \Delta(0)=$ $1.9 k_{B} T_{c}=h \times 7.9 \mathrm{GHz}, \ell=5.7 \mathrm{~nm}$. We chose the Larmor frequency $\omega / 2 \pi=1 \mathrm{MHz}$ and $\theta=0$. Note that Ref. [64] gives a mean free path larger by a factor of 4 and a smaller value of $\gamma$. For better comparison the normal metal is obtained by closing the superconducting energy gap and rescaling the plasma frequency. Gray dashed lines indicate the asymptotes to the superconductor. Losses due to the free space black body spectrum are not visible on this scale.

the approach of Mattis and Bardeen including disorder scattering [67], using the expressions of Pöpel [64]. The local limit recovers correctly the results from Refs. [68, 69]. For comparison we also give the curves for a fictitious normal metal, with the same parameters except that the gap is closed $(\Delta(0)=0)$. The description then coincides with the nonlocal BM model. The metallic plasma frequency was adjusted in order to take into account the redistribution of the spectral weight by disorder, as discussed in Refs. [69,70].

In the local regime $z \gg \ell$, the superconductor indeed shows a strong reduction of magnetic noise. This happens because the relevant frequency is below the gap, $\hbar \omega \ll \Delta(T)$, and magnetic fields are well screened by the Meissner effect. At large distances, we find good agreement with the expressions for the lossrate obtained by Skagerstam et al. [71] for a twofluid model, if the same re-scaled plasma frequency is taken into account (gray dashed asymptote in Fig. 3). In terms of the Green's tensor,

$$
\operatorname{Im} \mathcal{H}_{x x}(z, \omega) \approx \frac{\mu_{0} \lambda_{L}^{3}(T)}{4 \pi \delta^{2}(\omega, T) z^{4}}\left(\frac{3}{4}\right)^{2} .
$$

The Meissner-London length $\lambda_{L}(T)=\left(c / \omega_{p}\right) \Delta(0) / \Delta(T) \approx$ $\left(c / \omega_{p}\right)\left[1-\left(T / T_{c}\right)^{4}\right]^{-1}$ determines the penetration depth for quasistatic fields, while the skin depth $\delta(\omega, T)$ involves the conductivity for the normal fluid fraction. The Meissner effect becomes inefficient, however, if the spatial scale $1 / p$ of the noise field becomes comparable or smaller than the penetration depth $\lambda_{L}(T)$. The loss rate for $z \ll \lambda_{L}(T)$ then approaches the $1 / z$ asymptote of a normal conductor, see Fig. 3. At shorter length scales $z \ll \ell$, we recover the logarithmic scaling law found before for the normal conductor (thin dashed line). This illustrates the very general character of this regime that does not depend greatly on the material class.

\section{Lateral coherence}

A nonlocal conductivity creates spatial correlations in the current fluctuations below the surface which reduce the overall magnetic noise level. It is to be expected that this leaves also a signature in the correlations of the field. These correlations are universal for blackbody radiation [36] and have been studied in Refs. [38,-40] for homogeneous media and in Refs. [41-46] for the near field of bodies. The spatial correlation length can be much larger or much smaller than the wavelength, depending on the polariton modes that dominate the electromagnetic field noise. We find in this section that the correlation length is connected to the mean free path as a direct consequence of the ballistic motion of the charge carriers. This should be contrasted to electric fields near nonlocal solids where the spatial correlations were found to differ from the local description only at distances comparable to the Thomas-Fermi length $\Lambda$, with the mean free path $\ell$ not playing any role [44].

We are interested in the correlation between fields at a fixed height $z$ from the surface and laterally separated by a distance $\rho$. We define the coherence function as the cross-correlation spectrum of the normally ordered field operators in frequency space:

$$
\mathcal{B}_{i j}(\rho, z, \omega)=\int d t\left\langle: B_{i}(\rho, z, t) B_{j}(0, z, 0):\right\rangle \mathrm{e}^{\mathrm{i} \omega t} .
$$

The fluctuation-dissipation theorem for normally ordered operator products [72] provides the link to the two-point Green's tensor

$$
\mathcal{B}_{i j}(\rho, z, \omega)=2 \hbar \bar{n} \operatorname{Im} \mathcal{H}_{i j}\left(\mathbf{r}, \mathbf{r}^{\prime}, \omega\right),
$$

where the points $\mathbf{r}, \mathbf{r}^{\prime}$ are located at the same height and laterally separated by $\rho$. This generalizes Eq. (2). A general integral form is given in Appendix A. We drop the frequency arguments for simplicity in the following.

\subsection{Local limit}

Where a local description of the metal is sufficient, the coherence function in the near field can be evaluated asymptotically by expanding all integrands for values $p \gg 1 / \delta, \omega / c$. The resulting integrals have the form $\int_{0}^{\infty} d p e^{-2 p z} J_{n}(p \rho) p^{m}$, $\left(n, m \in \mathbb{N}_{0}\right.$ ) and can be evaluated exactly.

We find that at distances $\ell \ll z \ll \delta$, the magnetic coherence tensor depends on the distance $d=\sqrt{\rho^{2}+(2 z)^{2}}$ between one observation point and the mirror image of the other. The tensor elements are very well approximated by (see Fig. 4)

$$
\mathcal{B}_{i j}(\rho, z)=\frac{4 z^{2} \mathcal{B}_{x x}(0, z)}{d(d+2 z)}\left(\begin{array}{ccc}
2 & & \rho / z \\
& d / z & \\
\rho / z & 2+d / z
\end{array}\right) .
$$

Here, the noise spectrum $\mathcal{B}_{x x}(0, z)$ for $\rho=0$ was introduced as a convenient scale [see Eq. [6] ]. The axes are chosen such that the $x$-axis points along the separation between the two observation points. These quantities are independent of the specific material properties and depend only on the ratio $\rho / z$, i.e. the 
geometry of the system. An equivalent form for the $x x$ component was already given in Eq. (33) of Ref. [28], see also Ref. [2]. Note that the $x z$ cross-correlation was missed in Ref. [45].

The coherence functions decay on a typical length scale. For example, the $z z$-component (and similarly for the other ones) is characterized by the correlation length

$$
\ell \ll z: \quad \Delta_{z z}^{\mathrm{loc}}=2 \sqrt{3} z
$$

where $\mathcal{B}_{z z}(\rho, z)$ drops to half its value at $\rho=0$ (see Fig 4 a)). The approximate forms of Eq. (22) are not valid far beyond the correlation length where some correlation functions become negative, as shown in Fig. 4p). The agreement with the asymptotes is so high, however, that the curves are hardly distinguishable. The peak in the crossed $x z$-correlation arises from light paths that are reflected from the surface at oblique angles and whose fields are polarized in the $x z$-plane.

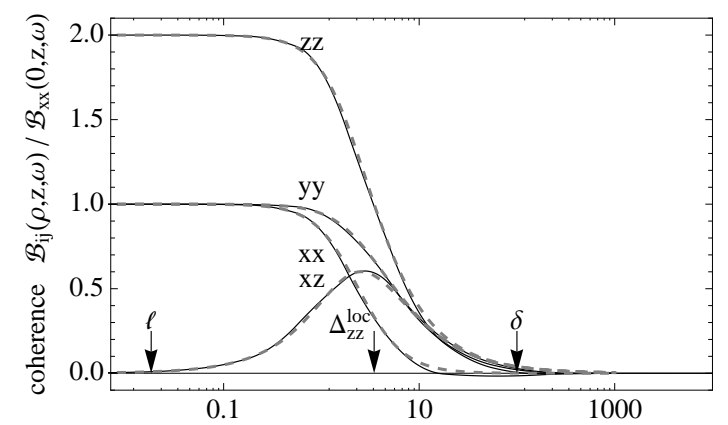

a)

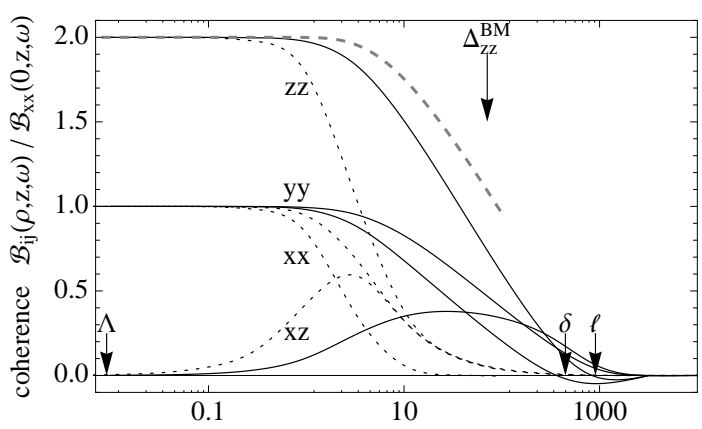

b)

distance $\rho / \mathrm{z}$

Figure 4. Components of the coherence tensor of the magnetic field near a gold surface. Solid (dotted) lines: Boltzmann-Mermin model with specular boundary condition (Drude model). a) Distance $z=$ $1 \mu \mathrm{m}$ much larger than mean free path $\ell=18 \mathrm{~nm}$ : all curves coincide with the local approximation (22) (dashed gray curves). b) Nonlocality becomes important at short distances. For better visibility we set $\ell=10 \mu \mathrm{m} \gg z=10 \mathrm{~nm}$ by lowering the scattering rate $\gamma$. The thick dashed line gives the rather coarse approximation from Eq. 28 (dashed gray curve). Frequency $\omega / 2 \pi=1 \mathrm{MHz}$.

\subsection{Nonlocal metal}

In the near field $z \ll \ell$, nonlocality leads to a larger coherence length than predicted by the local scenario, as is clearly visible in Fig. 4p).
In this section we extract the relevant scales by evaluating the two-point Green's tensor $\mathcal{H}_{z z}(\rho, z)$. It is necessary to interpolate the reflection coefficient $r_{\mathrm{s}}(p)$ between the local and the nonlocal limits to avoid an unphysical logarithmic divergence at the lower bound. The simplest choice is the Padé approximation

$$
\operatorname{Im} r_{\mathrm{s}}(p) \approx \frac{\operatorname{Im} r_{\mathrm{s}}^{\mathrm{loc}}(p)}{1+p \ell / 2} \approx \frac{1}{2 \delta^{2} p^{2}(1+p \ell / 2)},
$$

where the last form is appropriate for $p \gg 1 / \delta$ and the small- $p$ divergence is removed by the factor $p^{2}$ under the integral [see, e.g. Eq. [13]]. The integrals then give

$$
\begin{aligned}
& \mathcal{H}_{z z}(0, z) \approx \frac{\mu_{0}}{4 \pi \delta^{2} \ell} \mathrm{E}_{1}(4 z / \ell) \mathrm{e}^{4 z / \ell} \\
& \mathcal{H}_{z z}(\rho, z) \approx \frac{\mu_{0}}{8 \pi \delta^{2}} \int_{0}^{\infty} d p \frac{\exp (-2 p z) J_{0}(p \rho)}{1+p \ell / 2}
\end{aligned}
$$

where $E_{1}$ is the exponential integral defined after Eq. (15). We split the integral at $p=x_{0} / \rho$ where $x_{0} \approx 2.356$ is the first zero of the Bessel function $J_{0}(x)$. For $0<p<x_{0} / \rho, J_{0}(p \rho)$ is replaced by a spline $j(x)=1+b x^{2}+a x^{3}$, and replaced by its asymptote $j(x) \approx \sqrt{2 / \pi x} \cos (\pi / 4-x)$ for larger arguments. It turns out that the first interval gives the dominant contribution, since the oscillations beyond $x_{0} / \rho$ provide a cut-off for the integrand. We obtain

$$
\begin{aligned}
\mathcal{H}_{z z}(\rho, z) \approx & \mathcal{H}_{z z}(0, z)-\frac{\mu_{0}}{16 \pi \delta^{2} \ell}\left[-4 \mathrm{E}_{1}\left(2 x_{0} z / \rho\right)\right. \\
& +\frac{b \rho^{2}}{z^{2}}\left(1-\left(1+2 x_{0} z / \rho\right) \mathrm{e}^{-2 x_{0} z / \rho}\right) \\
& +\frac{a \rho^{3}}{z^{3}}\left(1-\left(1+2 x_{0} z / \rho+2\left(x_{0} z / \rho\right)^{2}\right) \mathrm{e}^{-2 x_{0} z / \rho}\right) \\
& \left.+\mathcal{O}\left(\frac{\rho}{\ell}, \frac{z}{\ell}\right)\right] .
\end{aligned}
$$

A careful glance at this expression shows that in the regime $z \ll \rho \ll \ell$, the first line dominates. The decay of the lateral coherence is therefore logarithmically (see Fig.4), as the smallargument approximation to the exponential integral illustrates (thick dashed line in Fig $4 \mathrm{p}$ ))

$$
\frac{\mathcal{B}_{z z}^{\mathrm{BM}}(\rho, z)}{\mathcal{B}_{x x}^{\mathrm{BM}}(0, z)} \approx 2-\frac{2 \mathrm{E}_{1}\left(2 x_{0} z / \rho\right)}{\mathrm{E}_{1}(4 z / \ell)} \approx 2-\frac{\ln \left[\left(2 x_{0} z / \rho\right)^{2}\right]}{\ln [4 z / \ell]} .
$$

This yields a coherence length

$$
z \ll \ell: \quad \Delta_{z z}^{\mathrm{BM}}=x_{0} \sqrt{z \ell}
$$

much larger than its local counterpart 23). The spatial correlations of magnetic near fields are therefore linked to the characteristic mean free path of ballistic transport. We recall that a similar discussion in Ref. [44] for electric correlations did not find Eq. 29) involving the mean free path $\ell$, but rather the Thomas-Fermi screening length $\Lambda$ which is typically smaller. The impact of spatial dispersion is therefore somewhat easier to reveal by analyzing magnetic fields. 


\section{Discussion}

We have found that the scattering mean free path of charge carriers sets the distance scale for the onset of nonlocal effects in the near field noise. For evanescent modes that dominate the near field, the fraction of the metallic volume that contributes to noise is limited by screening more efficiently than by the normal skin effect. The present calculation indicates, therefore, that loss rates are actually lower at short distances than predicted by local conductivity models (Ohm's law). This noise reduction sets in at atom-surface separations comparable to or below the mean free path. In a clean (crystalline) metal $\ell$ may take values in the order of $1 \mu \mathrm{m}$ which is at the limits of the experimentally accessible region (cf. the gray box in Fig. 1): typical traps operate at distances of $1 \ldots 100 \mu \mathrm{m}$ from the surface and can resolve lifetimes up to $10^{-2} \ldots 1 \mathrm{~s}$. The data shown in Fig. 1 are calculated at room temperature. Since both the conductivity and the mean free path depend on temperature, it is worth investigating whether the lifetimes of magnetic levels may be tuned by cooling the atom chip device. This strategy is hitting a limit in the extreme near field (distance $z \ll \ell$ ): the noise power becomes independent of the scattering rate of carriers (the Drude parameter $\gamma$ ), and also the details of the scattering mechanism of charge carriers at the inner surface become irrelevant. The near field spectrum [given in Eq. [15] ] has a rather general character and is expected to apply to doped semiconductors and even to superconductors, as our numerical calculations show (Fig. 3 ).

We have also analyzed the asymptotic form of the noise correlations in the short-distance range and found that magnetic fields are laterally coherent on a scale $\sim \sqrt{(\ell+z) z}$. This implies for an atom chip environment that fluctuating forces due to magnetic field gradients are smaller (their spectral density scales roughly with the inverse square of the correlation length). Also when matter-wave interferometry involves the spatial splitting of a thermal cloud or condensate, the increase in spatial coherence makes the device more robust against decoherence from magnetic noise (see [28, 73, 74]). The increase in spatial coherence intimately relates to the reduction of heat transfer via fluctuating near fields because the effective number of channels is inversely proportional to the "coherence area". For a more detailed discussion of this link, see Refs. [75, 76].

Patch potentials due to adsorbates on the surface are known to add significantly to the electric field noise, relevant for ion traps [77, 78] and systems involving precisely tuned electric dipole transitions, such as Rydberg states [79,-81]. Yet they will not effect the magnetic case. Static patches have no impact on the magnetic noise spectrum and magnetic surface-dipoles due to adsorbed atoms have only minor impact: A static charge trapped at a distance of $1 \mu \mathrm{m}$ from an atomic-scale electric dipole results in an interaction energy in the order of $20 \mathrm{MHz}$, while the interaction between two such dipoles gives only $1 \mathrm{kHz}$, and the magnetic counterpart for two magnetic dipoles of one Bohr magneton $\mu_{B}$ at the same distance gives $1 \mathrm{mHz}$. More prominent sources of magnetic noise might involve diffusive currents confined to a surface layer. Their effect may still be negligible, however, as the analysis of Ref. [82] has found.

All of these results imply that nonlocality may be visible at the edge of what is feasible with atom chips. Still, operating a chip trap at short distances is fundamentally limited by the
Casimir-Polder interaction that deforms and breaks the trapping potentials. Alternative setups might, therefore, address the broadening of magnetic transitions spectroscopically, e.g. using evanescent-wave based surface traps as in Ref. [83] or optical tweezers. One may also think of muonic or nuclear magnetic moments, as used in the experiment of Ref. [84] on spatial dispersion in superconductors.

Acknowledgments. We would like to thank B. Horovitz, F. Intravaia, J. Schiefele, and S. Slama for helpful discussions. Partial financial support from the German-Israeli Foundation for Scientific Research and Development (GIF), from the European Science Foundation (ESF 'Casimir-network'), and from the Deutsche Forschungsgemeinschaft (DFG) is acknowledged. We thank B. D'Anjou for implementing a numerical model of superconductors during an internship funded by Deutscher Akademischer Austauschdienst (DAAD-RISE).

\section{A The magnetic Green's tensor}

The Green's tensor describes the magnetic field

$$
B_{i}(\mathbf{r}, \omega)=\mathcal{H}_{i j}\left(\mathbf{r}, \mathbf{r}^{\prime}, \omega\right) \mu_{j}(\omega)
$$

radiated by a point-like magnetic dipole source placed in $\mathbf{r}^{\prime}$. Near a single surface, this field consists of a free-space part and a reflected contribution, and therefore $\mathcal{H}=\mathcal{H}^{F}+\mathcal{H}^{R}$.

For coinciding spatial arguments, the imaginary part of the free space Green's tensor is given by

$$
\operatorname{Im} \mathcal{H}_{i j}^{F}(\mathbf{r}, \mathbf{r}, \omega)=\frac{\mu_{0} \omega^{3}}{6 \pi c^{3}} \delta_{i j} .
$$

A detailed discussion including regularization procedures is given in Refs. [85, 86]. The general expression for the reflected Green's tensor reads

$$
\mathcal{H}^{R}\left(\mathbf{r}, \mathbf{r}^{\prime}, \omega\right)=\frac{\mu_{0}}{4 \pi} \int_{0}^{\infty} \frac{p d p}{\kappa} e^{-\kappa\left|z+z^{\prime}\right|}\left[r_{\mathrm{s}}(\omega, p) \boldsymbol{P}+r_{\mathrm{p}}(\omega, p) \boldsymbol{S}\right],
$$

where $\kappa=\sqrt{p^{2}-\omega^{2} / c^{2}}, \operatorname{Re} \kappa \geq 0, \operatorname{Im} \kappa \leq 0$, is the propagation constant. The tensor structure is included in

$$
\begin{aligned}
\boldsymbol{P} & =\left(\begin{array}{ccc}
\kappa^{2}\left(J_{0}(p \rho)-\frac{J_{1}(p \rho)}{p \rho}\right) & 0 & -p \kappa J_{1}(p \rho) \\
0 & \kappa^{2} \frac{J_{1}(p \rho)}{p \rho} & 0 \\
p \kappa J_{1}(p \rho) & 0 & p^{2} J_{0}(p \rho)
\end{array}\right) \\
\boldsymbol{S} & =\frac{\omega^{2}}{c^{2}}\left(\begin{array}{ccc}
\frac{J_{1}(p \rho)}{p \rho} & 0 & 0 \\
0 & J_{0}(p \rho)-\frac{J_{1}(p \rho)}{p \rho} & 0 \\
0 & 0 & 0
\end{array}\right),
\end{aligned}
$$

where $J_{n}(x)$ indicate Bessel functions of the first kind. The relative separation along the surface has length $\rho=\left[\left|\mathbf{r}-\mathbf{r}^{\prime}\right|^{2}-\right.$ $\left.\left|z-z^{\prime}\right|^{2}\right]^{1 / 2}$ and points along the $x$-axis. In the limit $\mathbf{r} \rightarrow \mathbf{r}^{\prime}$, we obtain the one-point reflected Green's tensor (3) that depends only on the distance from the surface. It is easy to check that the free-space contribution 31) is typically negligible at distances from a conducting surface smaller than the vacuum wavelength $c / \omega$, and we can drop the superscript ${ }^{R}$. 


\section{B Nonlocal reflectivities}

\section{B.1 Reflection coefficients}

For $p, s$-polarized incident waves, reflectivities are given by $[10]$

$$
r_{\mathrm{p}}=\frac{Z_{p}^{0}-Z_{p}}{Z_{p}^{0}+Z_{p}}, \quad r_{\mathrm{s}}=\frac{Z_{s}-Z_{s}^{0}}{Z_{s}+Z_{s}^{0}}
$$

where the surface impedances $Z_{s}, Z_{p}$ are made dimensionless by normalizing to the impedance of free space, $\tilde{Z}=\sqrt{\mu_{0} / \varepsilon_{0}}$. The well known results from local theory are [19,87]

$$
Z_{p}^{\mathrm{loc}}=\frac{\sqrt{\varepsilon(\omega) \omega^{2} / c^{2}-p^{2}}}{\varepsilon(\omega) \omega / c}, \quad Z_{s}^{\mathrm{loc}}=\frac{\omega / c}{\sqrt{\varepsilon(\omega) \omega^{2} / c^{2}-p^{2}}},
$$

where $\varepsilon(\omega)$ is the dielectric function (we set $\mu(\omega)=1$ ), and the reflectivities (32) reduce to Fresnel formulas. The surface impedance on the vacuum side, $Z_{p}^{0}, Z_{s}^{0}$, is obtained by setting $\varepsilon(\omega)=1$. Our focus is on metals at low frequencies where in the local limit, $\varepsilon(\omega) \omega^{2} / c^{2} \approx 2 \mathrm{i} / \delta^{2}(\omega)$ in terms of the skin depth (7), and on the sub-wavelength limit $p \gg \omega / c$. This gives an $r_{\mathrm{s}}$ coefficient

$$
r_{\mathrm{s}}^{\mathrm{loc}}(\omega, p) \approx \frac{\mathrm{i} p \delta(\omega)-\sqrt{2 \mathrm{i}-p^{2} \delta^{2}(\omega)}}{\mathrm{i} p \delta(\omega)+\sqrt{2 \mathrm{i}-p^{2} \delta^{2}(\omega)}} .
$$

The large-momentum asymptote $(p \gg 1 / \delta)$ is $r_{\mathrm{s}}(\omega, p) \rightarrow$ i $/\left(2 p^{2} \delta^{2}\right)$.

\section{B.2 Impedances for specular and diffuse scattering}

The additional boundary condition that charge carriers undergo specular reflection at the inner metal surface can be exploited to extend the conducting half-space into a fictitious homogeneous medium, using a similar symmetry for the fields [4, 7, 10]. The resulting impedances are

$$
\begin{aligned}
Z_{s}^{\text {spec }}(\omega, p)= & \frac{2 i \omega}{c \pi} \int_{0}^{\infty} d q \frac{1}{\varepsilon_{t}(\omega, \mathbf{k}) \omega^{2} / c^{2}-k^{2}} \\
Z_{p}^{\text {spec }}(\omega, p)= & \frac{2 i \omega}{c \pi} \int_{0}^{\infty} \frac{d q}{k^{2}}\left[\frac{p^{2}}{\varepsilon_{l}(\omega, \mathbf{k}) \omega^{2} / c^{2}}+\right. \\
& \left.+\frac{q^{2}}{\varepsilon_{t}(\omega, \mathbf{k}) \omega^{2} / c^{2}-k^{2}}\right],
\end{aligned}
$$

where the medium wave vector is $k^{2}=p^{2}+q^{2}$. The dielectric functions are $\varepsilon_{t, l}=\varepsilon_{b}+\mathrm{i} \sigma_{t, l} / \varepsilon_{0} \omega$ with a background polarization $\varepsilon_{b}$ that drops out in our regime. If the medium is local, $\varepsilon_{t, l}$ do not depend on $k$, and a direct calculation of the integrals brings us back to the Fresnel impedances (33).

The diffuse reflection of conduction electrons at the surface has been considered in Refs. [4, 11, 12, 88]. We have used the s-polarized impedance obtained in Ref. [12] where the additional boundary condition is implemented via the so-called dielectric approximation. This means that in the basic linear current-field relation [Eq. [1]], the volume integral is restricted to the medium-filled half-space alone. Solving a Wiener-Hopf equation that follows from the Maxwell equations, one gets an impedance

$$
Z_{s}^{\text {diff }}(\omega, p)=\frac{\mathrm{i} \pi \omega}{c}\left(\int_{0}^{\infty} \mathrm{d} q \log \left[\frac{q^{2}}{k^{2}-\varepsilon_{t}(\omega, \mathbf{k}) \omega^{2} / c^{2}}\right]\right)^{-1} \text { (37. }
$$

This recovers correctly the local limit at all values of $p$. However, this is not true for the p-polarized impedance given in the same work and used in the numerical evaluation in Fig. 2. The violation of charge conservation at the surface in the dielectric approximation has been discussed in Refs. [12-14]. We continue here with the specular boundary condition.

\section{B.3 Limiting behavior of the reflectivities}

For a metal with specular reflection of charge carriers, Eq. 35. is rewritten in a dimensionless form by rescaling the wavevector $k=\sqrt{p^{2}+q^{2}}=p t$. Making the low-frequency approximation described before Eq. (34), the non-local dielectric function becomes [see Eqs. (7, 9)]

$$
\varepsilon_{t}(\omega, \mathbf{k}) \approx \frac{2 \mathrm{i} f_{t}(0, \mathrm{i} / k \ell)}{\delta^{2}(\omega)}
$$

For simplicity, we drop in the following the frequency arguments and the redundant argument of the Lindhard function $f_{t}$. We are left with the integral

$$
\begin{aligned}
Z_{s}^{\text {spec }}(p) & =-\frac{2 i \omega}{\pi c} \int_{1}^{\infty} d t \frac{1}{\sqrt{t^{2}-1} p t\left[1-2 \mathrm{i} f_{t}(\mathrm{i} / p \ell t) /(p \delta t)^{2}\right]} \\
& \approx-\frac{2 i \omega}{\pi c} \int_{1}^{\infty} d t \frac{1+2 \mathrm{i} f_{t}(\mathrm{i} / p \ell t) /(p \delta t)^{2}}{\sqrt{t^{2}-1} p t} \\
& =-\frac{i \omega}{c p}+\frac{2 \omega / c}{p^{4} \delta^{2} \ell} .
\end{aligned}
$$

We expanded the denominator in the sub-skin depth regime $(p \gg 1 / \delta, 1 / \ell)$ and replaced, in the last step, the transverse Lindhard function [Eq. [11]] by its asymptote for large $p \ell$ :

$$
f_{t}(\mathrm{i} / p \ell t)=\frac{3 \pi}{4 p \ell t}+\mathcal{O}\left[(p \ell t)^{-2}\right] .
$$

This result complies with a similar calculation carried out in Ref. [89].

The first term in Eq. 39 corresponds to the free-space surface impedance in the sub-wavelength limit. The reflection amplitude 32, therefore becomes

$$
r_{\mathrm{s}}^{\mathrm{spec}}(p) \approx \frac{Z_{s}^{\mathrm{spec}}(p)+\mathrm{i} \omega /(c p)}{Z_{s}^{\mathrm{spec}}(p)-\mathrm{i} \omega /(c p)} \approx \frac{\mathrm{i}}{p^{3} \delta^{2} \ell}
$$

which gives Eq. (14).

In the scenario where charge carriers are reflected diffusely rather than specularly we start from Eq. 37). The same substitution of the integration variable gives

$$
Z_{s}^{\text {diff }}(p)=\frac{\mathrm{i} \pi \omega /(c p)}{\int_{1}^{\infty} \frac{d t t}{\sqrt{t^{2}-1}} \log \left[\frac{t^{2}-1}{t^{2}-2 \mathrm{i} f_{t}(\mathrm{i} / p \ell t) /(p \delta t)^{2}}\right]}
$$


The integrand is expanded in $1 /(p \delta)^{2}$, and the integration can be performed explicitly

$$
\begin{aligned}
& \int_{1}^{\infty} \frac{d t t}{\sqrt{t^{2}-1}} \log \left[\frac{t^{2}-1}{t^{2}-2 \mathrm{i}(p \delta t)^{-2} f_{t}(\mathrm{i} / p \ell t)}\right] \\
\approx & \int_{1}^{\infty} \frac{d t t}{\sqrt{t^{2}-1}}\left\{\log \left[\frac{t^{2}-1}{t^{2}}\right]+\frac{2 \mathrm{i} f_{t}(\mathrm{i} / p \ell t)}{(p \delta t)^{2}}\right\} \\
= & -\pi+\frac{3 \pi \mathrm{i}}{2 p^{3} \delta^{2} \ell} \quad \text { if } p \ell \gg 1 .
\end{aligned}
$$

The resulting impedance reads

$$
Z_{s}^{\text {diff }}(p) \approx-\frac{i \omega}{c p}+\frac{3 \omega / c}{2 p^{4} \delta^{2} \ell}
$$

This differs from Eq. (39) only by a factor $3 / 4$ in the second term, so that the reflection amplitude is smaller by this number compared to the specular boundary condition, Eq. (41).

\section{References}

1. H.B. Callen, T.A. Welton, Phys. Rev. 83(1), 34 (1951)

2. J. Nenonen, J. Montonen, T. Katila, Rev. Sci. Instr. 67(6), 2397 (1996)

3. J.A. Sidles, J.L. Garbini, W.M. Dougherty, S.H. Chao, Proc. IEEE 91(5), 799 (2003)

4. G. Reuter, E. Sondheimer, Proc. R. Soc. Series A, Math. Phys. pp. 336-364 (1948)

5. R.B. Dingle, Physica 19(1-12), 311 (1953)

6. J. Lindhard, Kgl. Danske Videnskab. Selskab. Mater.-Fys. Medd. 28(8), 1 (1954)

7. K. Kliewer, R. Fuchs, Phys. Rev. 172(3), 607 (1968)

8. K. Kliewer, R. Fuchs, Phys. Rev. 181(2), 552 (1969)

9. N. Mermin, Phys. Rev. B 1(5), 2362 (1970)

10. G.W. Ford, W.H. Weber, Phys. Rep. 113, 195 (1984)

11. K.L. Kliewer, R. Fuchs, Phys. Rev. B 2(8), 2923 (1970)

12. J. Foley, A. Devaney, Phys. Rev. B 12(8), 3104 (1975)

13. F. Flores, F. García Moliner, Introduction to the Theory of Solid Surfaces (Cambridge University Press, 1979)

14. F. Flores, F. García-Moliner, J. Physique 38(7), 863 (1977)

15. F. García-Moliner, F. Flores, J. Physique 38(7), 851 (1977)

16. P.J. Feibelman, Prog. Surf. Sci. 12(4), 287 (1982)

17. A. Pippard, Proc. R. Soc. Series A, Math. Phys. 191(1026), 385 (1947)

18. R. Chambers, Proc. R. Soc. Series A, Math. Phys. 215(1123), 481 (1952)

19. M. Dressel, G. Grüner, Electrodynamics of solids : optical properties of electrons in matter (Cambridge University Press, 2002)

20. A.I. Volokitin, B.N.J. Persson, Rev. Mod. Phys. 79(4), 1291 (2007)

21. P. Chapuis, M. Laroche, S. Volz, J. Greffet, Phys. Rev. B 77, 125402 (2008)

22. R. Podgornik, G. Cevc, B.B. Žekš, J. Chem. Phys. 87(10), 5957 (1987)

23. B. Jancovici, Phys. Rev. E 74, 052103 (2006)

24. B.E. Sernelius, Phys. Rev. B 71(23), 235114 (2005)

25. A.M. Contreras-Reyes, W.L. Mochan, Phys. Rev. A 72, 034102 (2005)

26. R. Esquivel-Sirvent, C. Villarreal, W.L. Mochán, A.M. ContrerasReyes, V.B. Svetovoy, J. Phys. A 39(21), 6323 (2006)
27. R. Folman, P. Krüger, J. Schmiedmayer, J. Denschlag, C. Henkel, Adv. At. Mol. Opt. Phy. 48, 263 (2002)

28. C. Henkel, P. Krüger, R. Folman, J. Schmiedmayer, Appl. Phys. B 76(2), 173 (2003)

29. J. Fortágh, C. Zimmermann, Rev. Mod. Phys. 79(1), 235 (2007)

30. J. Reichel, V. Vuletic, eds., Atom Chips (Wiley-VCH, Weinheim, 2011)

31. C. Henkel, M. Wilkens, Europhys. Lett. 47, 414 (1999)

32. C. Henkel, S. Pötting, M. Wilkens, Appl. Phys. B 69(5), 379 (1999)

33. M. Jones, C. Vale, D. Sahagun, B. Hall, E. Hinds, Phys. Rev. Lett. 91(8), 080401 (2003)

34. P.K. Rekdal, S. Scheel, P.L. Knight, E.A. Hinds, Phys. Rev. A 70(1), 013811 (2004)

35. D.B. Chklovskii, P.A. Lee, Phys. Rev. B 45(10), 5240 (1992)

36. L. Mandel, E. Wolf, Optical coherence and quantum optics (Cambridge University Press, Cambridge, 1995)

37. J.J. Greffet, C. Henkel, Contemp. Phys. 48(4), 183 (2007)

38. F. Gori, D. Ambrosini, V. Bagini, Opt. Commun. 107, 331 (1994)

39. S.A. Ponomarenko, E. Wolf, Phys. Rev. E 65, 016602 (2001)

40. K. Blomstedt, T. Setälä, A.T. Friberg, Phys. Rev. E 75, 026610 (2007)

41. R. Carminati, J.J. Greffet, Phys. Rev. Lett. 82, 1660 (1999)

42. C. Henkel, K. Joulain, R. Carminati, J.J. Greffet, Opt. Commun. 186, 57 (2000)

43. I. Dorofeyev, H. Fuchs, J. Jersch, Phys. Rev. E 65, 026610 (2002)

44. C. Henkel, K. Joulain, Appl. Phys. B 84(1), 61 (2006)

45. W. Lau, J. Shen, G. Veronis, S. Fan, Phys. Rev. E 76(1), 016601 (2007)

46. A. Norrman, T. Setälä, A.T. Friberg, J. Opt. Soc. Am. A 28(3), $391(2011)$

47. S.E. Boustani, P.R. Buenzli, P. Martin, Phys. Rev. E 73, 036113 (2006)

48. L. Samaj, B. Jancovici, Phys. Rev. E 78, 051119 (2008)

49. E.M. Purcell, Phys. Rev. 69(11-12), 674 (1946)

50. D.M. Harber, J.M. McGuirk, J.M. Obrecht, E.A. Cornell, J. Low Temp. Phys. 133, 229 (2003)

51. Y.j. Lin, I. Teper, C. Chin, V. Vuletić, Phys. Rev. Lett. 92(5), 050404 (2004)

52. H. Haakh, F. Intravaia, C. Henkel, S. Spagnolo, R. Passante, B. Power, F. Sols, Phys. Rev. A 80, 062905 (2009)

53. S.M. Rytov, Y.A. Kravtsov, V.I. Tatarskii, Principles of Statistical Radiophysics, Vol. 3, 4 (Springer, Berlin, 1989)

54. C. Henkel, K. Joulain, Europhys. Lett. 72, 929 (2005)

55. T. Varpula, T. Poutanen, J. Appl. Phys. 55(11), 4015 (1984)

56. G. Barton, Rep. Progr. Phys. 42(6), 963 (1979)

57. D. Bedeaux, J. Vlieger, Optical Properties of Surfaces (World Scientific, Singapore, 2004)

58. I.A. Larkin, M.I. Stockman, M. Achermann, V.I. Klimov, Phys. Rev. B 69(12), 121403 (2004)

59. V.A. Parsegian, Van der Waals Forces - A Handbook for Biologists, Chemists, Engineers, and Physicists (Cambridge University Press, New York, 2006)

60. L.D. Landau, E.M. Lifshitz, L.P. Pitaevskij, Course of theoretical physics. Vol. X: Physical kinetics. (Oxford, 1981)

61. D.B. Melrose, R.C. McPhedran, Electromagnetic processes in dispersive media (Cambridge University Press, Cambridge, 1991)

62. L. Weber, E. Gmelin, Appl. Phys. A 53(2), 136 (1991)

63. N.W. Ashcroft, N.D. Mermin, Solid state physics (Holt, Rinehart and Winston, New York, 1987)

64. R. Pöpel, J. Appl. Phys. 66(12), 5950 (1989)

65. P.B. Miller, Phys. Rev. 118(4), 928 (1960)

66. G. Rickayzen, Phys. Rev. 115(4), 795 (1959) 
67. D.C. Mattis, J. Bardeen, Phys. Rev. 111(2), 412 (1958)

68. W. Zimmermann, E. Brandt, M. Bauer, E. Seider, L. Genzel, Physica C 183(1-3), 99 (1991)

69. A.J. Berlinsky, C. Kallin, G. Rose, A.C. Shi, Phys. Rev. B 48(6), 4074 (1993)

70. G. Bimonte, H. Haakh, C. Henkel, F. Intravaia, J. Phys A 43(14), 145304 (2010)

71. B. Skagerstam, U. Hohenester, A. Eiguren, P. Rekdal, Phys. Rev. Lett. 97(7), 070401 (2006)

72. G.S. Agarwal, Phys. Rev. A 11(1), 230 (1975)

73. C. Cheng, M. Raymer, Phys. Rev. Lett. 82(24), 4807 (1999)

74. R. Fermani, S. Scheel, P.L. Knight, Phys. Rev. A 73, 032902 (2006)

75. S.A. Biehs, E. Rousseau, J.J. Greffet, Phys. Rev. Lett. 105, 234301 (2010)

76. P. Ben-Abdallah, K. Joulain, Phys. Rev. B 82, 121419(R) (2010)

77. Q. Turchette, B. King, D. Leibfried, D. Meekhof, C. Myatt, M. Rowe, C. Sackett, C. Wood, W. Itano, C. Monroe et al., Phys. Rev. A 61(6), 063418 (2000)

78. R. Dubessy, T. Coudreau, L. Guidoni, Phys. Rev. A 80(3), 031402 (2009)

79. J.D. Carter, J.D.D. Martin, Phy. Rev. A 83(3), 032902 (2011)

80. A. Tauschinsky, R. Thijssen, S. Whitlock, H. van den Heuvell, R. Spreeuw, Phys. Rev. A 81(6), 063411 (2010)

81. M.M. Müller, H.R. Haakh, T. Calarco, C.P. Koch, C. Henkel, arXiv:1104.2739 (2011)

82. C. Henkel, B. Horovitz, Phys. Rev. A 78(4), 042902 (2008)

83. H. Bender, P.W. Courteille, C. Marzok, C. Zimmermann, S. Slama, Phys. Rev. Lett. 104(8), 083201 (2010)

84. A. Suter, E. Morenzoni, R. Khasanov, H. Luetkens, T. Prokscha, N. Garifianov, Phys. Rev. Lett. 92(8), 087001 (2004)

85. P. de Vries, D. van Coevorden, A. Lagendijk, Rev. Mod. Phys. 70(2), 447 (1998)

86. C. Cohen-Tannoudji, J. Dupont-Roc, G. Grynberg, Photons and atoms (Intereditions, Paris, 1987)

87. J.D. Jackson, Classical electrodynamics (John Wiley and Sons Inc., New York, 1975)

88. P. Halevi, R. Fuchs, J. Phys. C 17, 3889 (1984)

89. R. Esquivel, V.B. Svetovoy, Phys. Rev. A 69(6), 062102 (2004) 